\title{
Some inconvenient truths
}

\section{R.P. Woodard}

Department of Physics, University of Florida, Gainesville, FL 32611, U.S.A.

E-mail: woodard@phys.ufl.edu

ABstract: A recent paper by Fröb employs the linearized Weyl-Weyl correlator to construct the tensor power spectrum. Although his purpose was to argue that infrared divergences and secular growth in the graviton propagator are gauge artefacts, a closer examination of the problem leads to the opposite conclusion. The analogies with the BMS symmetries of graviton scattering on a flat background, and with the Aharonov-Bohm effect of quantum mechanics, suggest that de Sitter breaking secular growth is likely to be observable in graviton loop effects. And a recent result for the vacuum polarization does seem to show it.

KEYwORDS: Cosmology of Theories beyond the SM, Models of Quantum Gravity, SpaceTime Symmetries

ARXIV EPRINT: 1506.04252 


\section{Contents}

1 Introduction 1

2 The power spectrum and TTS gauge 2

3 Facts are stubborn things 4

3.1 Derivatives moderate the IR, not invariance 4

3.2 Secular growth is distinct from IR divergences 5

3.3 Either Fourier transforms exist or not, pick one 6

3.4 Asymptotic symmetries are not fixed in flat space 6

3.5 Things couple to the metric, not the Weyl tensor $\quad 7$

4 Conclusion $\quad 7$

\section{Introduction}

There has been a long controversy about de Sitter breaking by the graviton propagator on de Sitter background [1-26]. Much has been learned that is valid no matter which view prevails. Significant insights include:

- A linearization instability precludes adding de Sitter invariant gauge fixing terms to the action, although enforcing de Sitter invariant exact gauge conditions as strong operator equations is allowed [13].

- The best way of expressing the tensor structure of a graviton propagator in an exact gauge is as a sum of differential projectors acting on scalar structure functions which obey simple equations $[15,23]$.

- The entire difference between the de Sitter invariant and de Sitter breaking constructions in the same fully-fixed gauge can be traced to what these scalar equations are and how one solves them. Further, this difference takes the form of a gauge transformation [24].

- A solution to the propagator equation need not correspond to a propagator in the sense of being the expectation value of the time-ordered product of two fields in the presence of some positive norm state $[27,28]$.

- Analytic continuation fails to register power law infrared divergences [14, 28, 29] and can convert positive into negative norm states [25]. 
A particularly important insight has been the close relation between the tensor power spectrum of primordial inflation and the graviton propagator in transverse-tracelesssynchronous (TTS) gauge, which poses a formidable problem for those who deny de Sitter breaking $[18,25]$. A recent paper by Fröb confronts this issue by using spatial Fourier transforms of the linearized Weyl-Weyl correlator to reconstruct the tensor power spectrum [30]. Because the linearized Weyl-Weyl correlator for de Sitter is infrared finite and free of secular dependence [7, 20, 22], Fröb argues that both of these features in the TTS gauge propagator are gauge artefacts. He further argues that one can consistently construct the tensor power spectrum using the infrared finite propagator obtained by Higuchi, Marolf and Morrison by subjecting the TTS gauge result to a coordinate transformation which grows at spatial infinity but preserves the TTS condition [17].

Fröb's attention to this problem is praise-worthy. However, a fuller examination of the facts leads to different conclusions, in particular:

- It is not invariance but derivatives which moderate infrared divergences;

- Infrared divergences are distinct from secular dependence;

- If the validity of spatial Fourier transforms is assumed then the linearized Weyl-Weyl correlator implies that the usual result for the graviton propagator is unique in TTS gauge;

- Conversely, if the validity of large coordinate transformations is asserted then the linearized Weyl-Weyl correlator does not provide a unique result for the tensor power spectrum;

- The fact that the symmetries of Bondi, van der Burg and Metzner [31] and Sachs [32] (hencforth BMS) in graviton scattering on flat background act nontrivially on Smatrix elements [33-35] seems to imply that we should not consider diffeomorphisms whose parameters grow at spatial infinity to be gauge transformations; and

- The analogy with the Aharonov-Bohm effect of quantum mechanics [36] suggests that secular dependence in the graviton propagator can have physical consequences, even though it takes the form of a gauge transformation of a completely gaugefixed quantity.

Section 2 of this paper reviews the TTS gauge propagator and its relation to the graviton power spectrum. Section 3 gives the arguments supporting the conclusions stated above. Section 4 summarizes the progress which has been made in the debate about de Sitter breaking.

\section{The power spectrum and TTS gauge}

The tensor power spectrum is extracted by taking the late time limit of the temporally coincident graviton 2-point function,

$$
\Delta_{h}^{2}(\eta, k) \equiv \frac{k^{3}}{2 \pi^{2}} \int d^{3} x e^{-i \vec{k} \cdot \vec{x}}\left\langle\Omega\left|h_{i j}(\eta, \vec{x}) h_{i j}(\eta, \overrightarrow{0})\right| \Omega\right\rangle,
$$


in the same TTS gauge that was long ago applied to cosmology by Lifshitz [37]. One defines the graviton field $h_{\mu \nu}(\eta, \vec{x})$ (also the Hubble parameter $H(\eta)$ and the first slow roll parameter $\epsilon(\eta))$ in open conformal coordinates,

$$
g_{\mu \nu} \equiv a^{2}\left[\eta_{\mu \nu}+h_{\mu \nu}\right] \quad, \quad H(\eta) \equiv \frac{a^{\prime}(\eta)}{a^{2}(\eta)} \quad, \quad \epsilon(\eta) \equiv-\frac{H^{\prime}}{a H^{2}}
$$

De Sitter corresponds to the special case of $\epsilon=0$ but I will work with general $\epsilon(\eta)$. One reaches TTS gauge by first imposing the volume gauge condition,

$$
F_{\mu}(\eta, \vec{x}) \equiv \eta^{\rho \sigma}\left[h_{\mu \rho, \sigma}-\frac{1}{2} \partial_{\mu} h_{\rho \sigma}+2 H a h_{\mu \rho} \delta_{\sigma}^{0}\right]=0 .
$$

Then the linearized Einstein equation, plus a complete exhaustion of all residual gauge freedom in spatial Fourier space to eliminate $h_{00}$ and $h_{0 i}$, allows one to express $h_{i j}$ as $[38,39]$,

$$
\begin{aligned}
h_{i j}(\eta, \vec{x})=\sqrt{32 \pi G} \int \frac{d^{3} k}{(2 \pi)^{3}} \sum_{\lambda= \pm 2}\left\{u(\eta, k) e^{i \vec{k} \cdot \vec{x}} \varepsilon_{i j}(\vec{k}, \lambda) \alpha(\vec{k}, \lambda)\right. & \\
& \left.+u^{*}(\eta, k) e^{-i \vec{k} \cdot \vec{x}} \varepsilon_{i j}^{*}(\vec{k}, \lambda) \alpha^{\dagger}(\vec{k}, \lambda)\right\} .
\end{aligned}
$$

The transverse-traceless polarization tensors $\varepsilon_{i j}(\vec{k}, \lambda)$ are identical to those of flat space, and the mode function $u(\eta, k)$ obeys the same equation as a massless, minimally coupled (MMC) scalar [37],

$$
u^{\prime \prime}+2 H a u^{\prime}+k^{2} u=0 \quad, \quad u u^{\prime *}-u^{\prime} u^{*}=\frac{i}{a^{2}} .
$$

The vacuum state obeys $\alpha(\vec{k}, \lambda)|\Omega\rangle=0$ and canonical quantization implies,

$$
\left[\alpha(\vec{k}, \lambda), \alpha^{\dagger}\left(\vec{k}^{\prime}, \lambda^{\prime}\right)\right]=\delta_{\lambda \lambda^{\prime}}(2 \pi)^{3} \delta^{3}\left(\vec{k}-\vec{k}^{\prime}\right) .
$$

Putting everything together gives a simple result for $\Delta_{h}^{2}(\eta, k)$,

$$
\Delta_{h}^{2}(\eta, k)=\frac{k^{3}}{2 \pi^{2}} \times 32 \pi G \times 2 \times|u(\eta, k)|^{2}=\frac{32}{\pi} G k^{3}|u(\eta, k)|^{2} .
$$

The TTS gauge propagator is,

$$
\begin{aligned}
\left.i{ }_{i j} \Delta_{k \ell}\right]\left(x ; x^{\prime}\right) & \equiv\left\langle\Omega\left|T\left[h_{i j}(\eta, \vec{x}) h_{k \ell}\left(\eta^{\prime}, \vec{x}^{\prime}\right)\right]\right| \Omega\right\rangle, \\
& =16 \pi G\left[\Pi_{i k} \Pi_{j \ell}+\Pi_{i \ell} \Pi_{j k}-\Pi_{i j} \Pi_{k \ell}\right] i \Delta\left(x ; x^{\prime}\right),
\end{aligned}
$$

where $\Pi_{i j} \equiv \delta_{i j}-\frac{\partial_{i} \partial_{j}}{\nabla^{2}}$ is the transverse projection operator and $i \Delta\left(x ; x^{\prime}\right)$ is the propagator for a MMC scalar,

$$
i \Delta\left(x ; x^{\prime}\right)=\int \frac{d^{3} k}{(2 \pi)^{3}} e^{i \vec{k} \cdot\left(\vec{x}-\vec{x}^{\prime}\right)}\left[\theta\left(\eta-\eta^{\prime}\right) u(\eta, k) u^{*}\left(\eta^{\prime}, k\right)+\theta\left(\eta^{\prime}-\eta\right) u^{*}(\eta, k) u\left(\eta^{\prime}, k\right)\right] .
$$


The relation between the power spectrum and the TTS propagator is,

$$
\begin{aligned}
\Delta_{h}^{2}(\eta, k) & =\frac{k^{3}}{2 \pi^{2}} \int d^{3} x e^{-i \vec{k} \cdot \vec{x}} \times i\left[_{i j} \Delta_{i j}\right](\eta, \vec{x} ; \eta, \overrightarrow{0}), \\
& =\frac{32}{\pi} G k^{3} \int d^{3} x e^{-i \vec{k} \cdot \vec{x}} i \Delta(\eta, \vec{x} ; \eta, \overrightarrow{0}) .
\end{aligned}
$$

Inverting relation (2.12) gives the coincident time MMC scalar propagator, and hence also the TTS gauge graviton propagator,

$$
i \Delta(\eta, \vec{x} ; \eta, \overrightarrow{0})=\int_{0}^{\infty} \frac{d k}{k} \frac{\Delta_{h}^{2}(\eta, k)}{64 \pi G} .
$$

No very explicit solution to (2.5) is known for general $\epsilon(\eta)$ [40] but the constant $\epsilon(\eta)$ solution is,

$$
\epsilon^{\prime}=0 \quad \Longrightarrow \quad u(\eta, k)=\sqrt{\frac{\pi}{4(1-\epsilon) H a^{3}}} H_{\nu}^{(1)}\left(\frac{k}{(1-\epsilon) H a}\right) \quad, \quad \nu \equiv \frac{1}{2}\left(\frac{3-\epsilon}{1-\epsilon}\right) .
$$

For the inflationary case of $0 \leq \epsilon<1$ the late time limiting form is,

$$
\epsilon^{\prime}=0 \quad \Longrightarrow \quad u(\eta, k) \longrightarrow-\frac{i \Gamma(\nu)}{\sqrt{4 \pi}}\left[(1-\epsilon) H a^{\epsilon}\right]^{\frac{1}{1-\epsilon}}\left(\frac{2}{k}\right)^{\nu}
$$

Expression (2.15) is also the limiting form for small $k$. Ford and Parker used this (and the limiting form for $\epsilon>1)$ to show that the mode sum for $i \Delta\left(x ; x^{\prime}\right)$ - and hence also for the TTS gauge propagator - is infrared divergent for all constant $\epsilon$ geometries in the range $0 \leq \epsilon \leq \frac{3}{2}[41]$.

Expression (2.2) implies $\partial_{0}\left[H a^{\epsilon}\right]=\epsilon^{\prime} H^{2} a^{1+\epsilon}$, so $H a^{\epsilon}$ is constant for constant $\epsilon$. It is usual to evaluate this constant at the time $\eta_{k}$ of first horizon crossing when $k=H\left(\eta_{k}\right) a\left(\eta_{k}\right)$,

$$
\epsilon^{\prime}=0 \quad \Longrightarrow \quad H(\eta)[a(\eta)]^{\epsilon}=H\left(\eta_{k}\right)\left[a\left(\eta_{k}\right)\right]^{\epsilon}=k^{\epsilon}\left[H\left(\eta_{k}\right)\right]^{1-\epsilon}
$$

Substituting relations (2.15) and (2.16) allows us to compute the late time limit of the tensor power spectrum for constant $\epsilon$,

$$
\epsilon^{\prime}=0 \quad \Longrightarrow \quad \Delta_{h}^{2}(\eta, k) \longrightarrow \frac{16}{\pi} G H^{2}\left(\eta_{k}\right) \times \frac{4 \Gamma^{2}(\nu)}{\pi}\left[2^{\epsilon}(1-\epsilon)\right]^{\frac{2}{1-\epsilon}} .
$$

\section{Facts are stubborn things}

In this section I marshal facts from the previous section to support my views on infrared divergences and de Sitter breaking secular dependence.

\subsection{Derivatives moderate the IR, not invariance}

It is not the fact that the linearized Weyl tensor is invariant which renders its 2-point function infrared finite for de Sitter, but rather the presence of derivatives. To see this, note from the small $k$ limiting form (2.15) that the infrared divergence of $i \Delta\left(x ; x^{\prime}\right)$ (and hence also the TTS gauge propagator) is only logarithmic for the de Sitter case of $\epsilon=0$. 
This means only a single derivative is needed to eliminate it. In particular, there is no infrared divergence in the noninvariant quantity,

$$
\frac{\partial}{\partial x^{m}} i\left[{ }_{i j} \Delta_{k \ell}\right]\left(x ; x^{\prime}\right)=\left\langle\Omega\left|T\left[h_{i j, m}(x) h_{k \ell}\left(x^{\prime}\right)\right]\right| \Omega\right\rangle .
$$

A single temporal derivative would also produce an infrared finite mode sum because the right hand side of expression (2.15) is constant.

It is not even true that invariance guarantees infrared finiteness for other constant values of $\epsilon$. From the small $k$ limiting form (2.15) one can see that the mode sum for the propagator goes like,

$$
i \Delta\left(x ; x^{\prime}\right) \sim \int d k k^{2} \times \frac{1}{k^{2 \nu}}=\int \frac{d k}{k}\left(\frac{1}{k}\right)^{\frac{2 \epsilon}{1-\epsilon}} .
$$

The linearized Weyl-Weyl correlator contains four derivatives, so its mode sum will be infrared divergent for any value of $\epsilon$ in the range $\frac{2}{3} \leq \epsilon \leq \frac{5}{4}$, with the upper limit derived by generalizing (2.15) to the decelerating case of $\epsilon>1$.

\subsection{Secular growth is distinct from IR divergences}

Infrared divergences (which only occur in open coordinates) are quite distinct from secular growth, which occurs even in closed coordinates [8]. Secular growth manifests in both coordinate systems because higher and higher modes approach the constant (2.15) at late times, which is not even different between the two coordinate systems for high modes [25]. The infrared has nothing at all to do with it, despite the fact that the late time limiting form (2.15) is the same as the limiting form for small $k$. Allen and Folacci [42] demonstrated the distinction for the discrete MMC scalar mode sum on closed coordinates by expunging the 0 -mode and finding precisely the secular growth previously obtained in open coordinates by Vilenkin and Ford [43], Linde [44] and by Starobinsky [45].

In contrast, infrared divergences are associated with large numbers of small $k$ modes, and with the initial conditions [46]. At any fixed open coordinate time there are an infinite number of super-horizon modes already near the limiting form (2.15), whereas the number of super-horizon modes is finite for any fixed closed coordinate time [25].

Note also that a single time derivative of the graviton propagator eliminates its IR divergence (on de Sitter) but not its secular growth. If one regulates the infrared problem on de Sitter so as to preserve homogeneity and isotropy then the MMC scalar propagator acquires an extra term involving $\ln \left[a(\eta) a\left(\eta^{\prime}\right)\right]$ [47]. It requires derivatives with respect to both coordinates to annihilate this contribution,

$$
\partial_{\mu} \ln \left[a(\eta) a\left(\eta^{\prime}\right)\right]=H a \delta_{\mu}^{0} \quad, \quad \partial_{\mu} \partial_{\nu}^{\prime} \ln \left[a(\eta) a\left(\eta^{\prime}\right)\right]=0
$$

Even more derivatives are needed to eliminate the extra terms which appear for larger values of $\epsilon<1[28,39]$. And differentiated propagators do matter: the secular growth experienced at one loop by massless fermions on de Sitter derives entirely from diagrams for which the graviton propagator is acted upon by a single derivative [48]. 


\subsection{Either Fourier transforms exist or not, pick one}

Either the spatial Fourier transform of $h_{i j}(t, \vec{x})$ exists or it does not, and neither possibility supports de Sitter invariance. A different conclusion is only possible by inconsistently using spatial Fourier transforms to convert the linearized Weyl-Weyl correlator into $\Delta_{h}^{2}(\eta, k)$ but not $i \Delta(\eta, \vec{x} ; \eta, \overrightarrow{0})$.

If the first case is accepted (spatial Fourier transforms exist) then the TTS conditions completely fix the gauge and the on-shell field redefinition of Higuchi, Marolf and Morrison [17] is not allowed. In that case the linearized Weyl-Weyl correlator can indeed be used to derive a unique result (2.7) for the tensor power spectrum, but it also gives a unique result (2.9)-(2.10) for the propagator, with the de Sitter breaking secular growth.

On the other hand, if the validity of spatial Fourier transforms is denied, then the spatial Fourier transform of the linearized Weyl-Weyl correlator does not give a unique result for the tensor power spectrum. It must be supplemented by some boundary condition at spatial infinity which defines how to invert $\nabla^{2} \rightarrow-k^{2}$. In that case the linearized WeylWeyl correlator does not contain all physical information about free gravitons, and there is no significance to the fact that this correlator fails to show the de Sitter breaking secular dependence of the TTS gauge propagator (2.9)-(2.10).

If spatial Fourier transforms do not exist one must also accept that the graviton field operator possesses degrees of freedom in addition to the creation and annihilation operators $\alpha(\vec{k})$ and $\alpha^{\dagger}(\vec{k})$. What Higuchi, Marolf and Morrison did instead is to retain only these degrees of freedom and reshuffle how $h_{i j}(t, \vec{x})$ depends upon them [17]. That corresponds to a noncanonical quantization, not a fixing of some residual gauge freedom [18].

Finally, it is important to note that there should be no local test to distinguish between the manifold $R^{3} \times R$, on which the existence of spatial Fourier transforms might be an issue, and the manifold $T^{3} \times R$, on which spatial Fourier transforms certainly exist. On the manifold $T^{3} \times R$ the TTS gauge propagator becomes a mode sum to which the continuum result (2.9)-(2.10) is an excellent approximation for spatial coordinate separations which are small compared to the $T^{3}$ radii. As pointed out above, this propagator will certainly exhibit the de Sitter breaking secular growth which is at issue.

\subsection{Asymptotic symmetries are not fixed in flat space}

The debate over de Sitter breaking sometimes engenders a sense of déjà vu. The issue of spatially growing coordinate redefinitions has already come up - quite a long time ago [31, 32 ] - in the context of graviton scattering amplitudes on flat space background. These BMS transformations act nontrivially on scattering amplitudes and are not considered to be gauge transformations [33-35]. In particular, no one employs them to alter the infrared behavior of the graviton propagator, which cannot be changed because it has physical consequences [50]. Instead the action of a BMS transformation on a classical configuration corresponds to the physical differences which prevail after a gravitational wave has passed [33]. Note that the linearized Weyl curvature vanishes before and after the passage of such a wave. 
It is difficult to discern any difference of principle between the BMS transformations of graviton scattering on flat background and the analogous spatially growing symmetries of cosmology worked out by Hinterbichler, Hui and Khoury [49], the first of which was exploited by Higuchi, Marolf and Morrison [17]. It would seem to follow that these transformations should not be regarded as gauge symmetries, nor should they be employed to alter the infrared properties of the graviton propagator. The same conclusion follows from working on the spatial manifold $T^{3} \times R$, which has no extra symmetries.

\subsection{Things couple to the metric, not the Weyl tensor}

Another debate physics has already seen is whether or not undifferentiated gauge fields can mediate physical effects when the field strength vanishes. It was obvious from the first charged particle wave equations of the 1920's that matter fields do not couple to the electromagnetic field strength tensor $F_{\mu \nu}$ but rather to the undifferentiated vector potential $A_{\mu}$. Hence there must sometimes be electromagnetic effects in regions throughout which the field strength vanishes. But physicists are as prone to feel prejudice as any other humans, and this obvious conclusion was denied for decades. Then came the work of Ehrenberg and Siday [51], followed by that of Aharonov and Bohm [36]. Everyone knows how the experiment turned out [52].

The analogy to gravity seems obvious. The fact that the secular growth of the TTS propagator (2.9)-(2.10) takes the form of a linearized gauge transformation (in a completely gauge-fixed result) explains why it drops out of the linearized Weyl-Weyl correlator. However, that does not mean this time dependence is unphysical, any more than the vanishing electromagnetic field strength implies that the Aharonov-Bohm potential is unphysical. Matter, and gravity itself, couples to the metric, not to the linearized Weyl tensor, so there must be circumstances under which the metric can communicate physical effects even when the linearized Weyl tensor vanishes. One of these may already have been found in a recent one graviton loop computation of the vacuum polarization on de Sitter background (cf. section 3.5 of) [53].

\section{Conclusion}

The decades over which varying opinions have been expressed about de Sitter breaking in the graviton propagator $[1-26,30]$ might make the debate seem interminable. However, real progress has been made and a definitive consensus has been reached on a number of essential issues:

- All now agree on the validity of the Feynman rules used for all existing graviton loop computations on de Sitter [53-70];

- All now agree that the open and closed coordinate mode sums show the same secular growth;

- All now agree that it is valid to regard de Sitter as a special case of inflationary cosmology in open coordinates; 
- The crucial importance of $\Delta_{h}^{2}(\eta, k)$ has been recognized; and

- The significance of large scale transformations has been recognized.

This has been achieved by members of the different communities thoughtfully considering each other's arguments. Fröb's study makes a fine addition, and I have tried to reply in the same spirit.

Through Morrison's work we can now quantify the rather small differences between the de Sitter invariant and breaking propagators [24]. These differences survive in the one graviton loop correction to the vacuum polarization (cf. section 3.5 of) [53]. What remains is to achieve a consensus on observables so that the physical significance of this result can be determined.

\section{Acknowledgments}

I am grateful for conversations and correspondence on this subject with A. Fröb, A Higuchi, S. P. Miao and G. Pimentel. This work was partially supported by NSF grants PHY1205591 and PHY-1506513, and by the Institute for Fundamental Theory at the University of Florida.

Open Access. This article is distributed under the terms of the Creative Commons Attribution License (CC-BY 4.0), which permits any use, distribution and reproduction in any medium, provided the original author(s) and source are credited.

\section{References}

[1] I. Antoniadis and E. Mottola, Graviton fluctuations in de Sitter space, J. Math. Phys. 32 (1991) 1037 [INSPIRE].

[2] B. Allen and M. Turyn, An evaluation of the graviton propagator in de Sitter space, Nucl. Phys. B 292 (1987) 813 [INSPIRE].

[3] N.C. Tsamis and R.P. Woodard, The structure of perturbative quantum gravity on a de Sitter background, Commun. Math. Phys. 162 (1994) 217 [InSPIRE].

[4] G. Kleppe, Breaking of de Sitter invariance in quantum cosmological gravity, Phys. Lett. B 317 (1993) 305 [INSPIRE].

[5] S.W. Hawking, T. Hertog and N. Turok, Gravitational waves in open de Sitter space, Phys. Rev. D 62 (2000) 063502 [hep-th/0003016] [INSPIRE].

[6] A. Higuchi and S.S. Kouris, The covariant graviton propagator in de Sitter space-time, Class. Quant. Grav. 18 (2001) 4317 [gr-qc/0107036] [INSPIRE].

[7] S.S. Kouris, The Weyl tensor two point function in de Sitter space-time, Class. Quant. Grav. 18 (2001) 4961 [Class. Quant. Grav. 29 (2012) 169501] [Erratum ibid. 29 (2012) 169501] [gr-qc/0107064] [INSPIRE].

[8] A. Higuchi and R.H. Weeks, The physical graviton two point function in de Sitter space-time with S3 spatial sections, Class. Quant. Grav. 20 (2003) 3005 [gr-qc/0212031] [INSPIRE].

[9] R.P. Woodard, De Sitter breaking in field theory, gr-qc/0408002 [INSPIRE]. 
[10] A. Higuchi and Y.C. Lee, How to use retarded Green's functions in de Sitter spacetime, Phys. Rev. D 78 (2008) 084031 [arXiv: 0808.0642] [INSPIRE].

[11] M. Faizal and A. Higuchi, On the FP-ghost propagators for Yang-Mills theories and perturbative quantum gravity in the covariant gauge in de Sitter spacetime, Phys. Rev. D 78 (2008) 067502 [arXiv:0806.3735] [INSPIRE].

[12] D. Marolf and I.A. Morrison, Group averaging for de Sitter free fields, Class. Quant. Grav. 26 (2009) 235003 [arXiv:0810.5163] [InSPIRE].

[13] S.P. Miao, N.C. Tsamis and R.P. Woodard, Transforming to Lorentz gauge on de Sitter, J. Math. Phys. 50 (2009) 122502 [arXiv:0907.4930] [INSPIRE].

[14] S.P. Miao, N.C. Tsamis and R.P. Woodard, De Sitter breaking through infrared divergences, J. Math. Phys. 51 (2010) 072503 [arXiv: 1002.4037] [inSPIRE].

[15] S.P. Miao, N.C. Tsamis and R.P. Woodard, The graviton propagator in de Donder gauge on de Sitter background, J. Math. Phys. 52 (2011) 122301 [arXiv:1106.0925] [INSPIRE].

[16] M. Faizal and A. Higuchi, Physical equivalence between the covariant and physical graviton two-point functions in de Sitter spacetime, Phys. Rev. D 85 (2012) 124021 [arXiv:1107.0395] [INSPIRE].

[17] A. Higuchi, D. Marolf and I.A. Morrison, De Sitter invariance of the dS graviton vacuum, Class. Quant. Grav. 28 (2011) 245012 [arXiv:1107.2712] [INSPIRE].

[18] S.P. Miao, N.C. Tsamis and R.P. Woodard, Gauging away physics, Class. Quant. Grav. 28 (2011) 245013 [arXiv:1107.4733] [inSPIRE].

[19] E.O. Kahya, S.P. Miao and R.P. Woodard, The coincidence limit of the graviton propagator in de Donder gauge on de Sitter background, J. Math. Phys. 53 (2012) 022304 [arXiv:1112.4420] [INSPIRE].

[20] P.J. Mora and R.P. Woodard, Linearized Weyl-Weyl correlator in a de Sitter breaking gauge, Phys. Rev. D 85 (2012) 124048 [arXiv:1202.0999] [inSPIRE].

[21] A. Higuchi, Equivalence between the Weyl-tensor and gauge-invariant graviton two-point functions in Minkowski and de Sitter spaces, arXiv:1204.1684 [INSPIRE].

[22] P.J. Mora, N.C. Tsamis and R.P. Woodard, Weyl-Weyl correlator in de Donder gauge on de Sitter, Phys. Rev. D 86 (2012) 084016 [arXiv:1205.4466] [InSPIRE].

[23] P.J. Mora, N.C. Tsamis and R.P. Woodard, Graviton propagator in a general invariant gauge on de Sitter, J. Math. Phys. 53 (2012) 122502 [arXiv:1205.4468] [INSPIRE].

[24] I.A. Morrison, On cosmic hair and "de Sitter breaking" in linearized quantum gravity, arXiv: 1302.1860 [INSPIRE].

[25] S.P. Miao, P.J. Mora, N.C. Tsamis and R.P. Woodard, Perils of analytic continuation, Phys. Rev. D 89 (2014) 104004 [arXiv: 1306.5410] [InSPIRE].

[26] R.P. Bernar, L.C. Crispino and A. Higuchi, Infrared-finite graviton two-point function in static de Sitter space, Phys. Rev. D 90 (2014) 024045 [arXiv: 1405.3827] [INSPIRE].

[27] N.C. Tsamis and R.P. Woodard, Schwinger's propagator is only a Green's function, Class. Quant. Grav. 18 (2001) 83 [hep-ph/0007167] [INSPIRE].

[28] T.M. Janssen, S.P. Miao, T. Prokopec and R.P. Woodard, Infrared propagator corrections for constant deceleration, Class. Quant. Grav. 25 (2008) 245013 [arXiv:0808.2449] [InSPIRE]. 
[29] A. Folacci, BRST quantization of the massless minimally coupled scalar field in de Sitter space: zero modes, euclideanization and quantization, Phys. Rev. D 46 (1992) 2553 [arXiv: 0911.2064] [INSPIRE].

[30] M.B. Fröb, The Weyl tensor correlator in cosmological spacetimes, JCAP 12 (2014) 010 [arXiv: 1409.7964] [INSPIRE].

[31] H. Bondi, M.G.J. van der Burg and A.W.K. Metzner, Gravitational waves in general relativity. 7. Waves from axisymmetric isolated systems, Proc. Roy. Soc. Lond. A 269 (1962) 21 [INSPIRE].

[32] R.K. Sachs, Gravitational waves in general relativity. 8. Waves in asymptotically flat space-times, Proc. Roy. Soc. Lond. A 270 (1962) 103 [InSPIRE].

[33] A. Strominger and A. Zhiboedov, Gravitational memory, BMS supertranslations and soft theorems, JHEP 01 (2016) 086 [arXiv:1411.5745] [INSPIRE].

[34] A. Strominger, On BMS invariance of gravitational scattering, JHEP 07 (2014) 152 [arXiv: 1312.2229] [INSPIRE].

[35] T. He, V. Lysov, P. Mitra and A. Strominger, BMS supertranslations and Weinberg's soft graviton theorem, JHEP 05 (2015) 151 [arXiv:1401.7026] [INSPIRE].

[36] Y. Aharonov and D. Bohm, Significance of electromagnetic potentials in the quantum theory, Phys. Rev. 115 (1959) 485 [inSPIRE].

[37] E. Lifshitz, On the gravitational stability of the expanding universe, J. Phys. (USSR) 10 (1946) 116 [INSPIRE].

[38] N.C. Tsamis and R.P. Woodard, Mode analysis and ward identities for perturbative quantum gravity in de Sitter space, Phys. Lett. B 292 (1992) 269 [INSPIRE].

[39] J. Iliopoulos, T.N. Tomaras, N.C. Tsamis and R.P. Woodard, Perturbative quantum gravity and Newton's law on a flat Robertson-Walker background, Nucl. Phys. B 534 (1998) 419 [gr-qc/9801028] [INSPIRE].

[40] N.C. Tsamis and R.P. Woodard, Plane waves in a general Robertson-Walker background, Class. Quant. Grav. 20 (2003) 5205 [astro-ph/0206010] [INSPIRE].

[41] L.H. Ford and L. Parker, Infrared divergences in a class of Robertson-Walker universes, Phys. Rev. D 16 (1977) 245 [INSPIRE].

[42] B. Allen and A. Folacci, The massless minimally coupled scalar field in de Sitter space, Phys. Rev. D 35 (1987) 3771 [inSPIRE].

[43] A. Vilenkin and L.H. Ford, Gravitational effects upon cosmological phase transitions, Phys. Rev. D 26 (1982) 1231 [INSPIRE].

[44] A.D. Linde, Scalar field fluctuations in expanding universe and the new inflationary universe scenario, Phys. Lett. B 116 (1982) 335 [INSPIRE].

[45] A.A. Starobinsky, Dynamics of phase transition in the new inflationary universe scenario and generation of perturbations, Phys. Lett. B 117 (1982) 175 [INSPIRE].

[46] S.A. Fulling, M. Sweeny and R.M. Wald, Singularity structure of the two point function in quantum field theory in curved space-time, Commun. Math. Phys. 63 (1978) 257 [INSPIRE].

[47] V.K. Onemli and R.P. Woodard, Superacceleration from massless, minimally coupled phi** 4 , Class. Quant. Grav. 19 (2002) 4607 [gr-qc/0204065] [inSPIRE]. 
[48] S.-P. Miao and R.P. Woodard, A simple operator check of the effective fermion mode function during inflation, Class. Quant. Grav. 25 (2008) 145009 [arXiv:0803.2377] [INSPIRE].

[49] K. Hinterbichler, L. Hui and J. Khoury, An infinite set of Ward identities for adiabatic modes in cosmology, JCAP 01 (2014) 039 [arXiv: 1304.5527] [INSPIRE].

[50] S. Weinberg, Infrared photons and gravitons, Phys. Rev. 140 (1965) B516 [InSPIRE].

[51] W. Ehrenberg and R.E. Siday, The refractive index in electron optics and the principles of dynamics, Proc. Phys. Soc. B 62 (1948) 8.

[52] R.G. Chambers, Shift of an electron interference pattern by enclosed magnetic flux, Phys. Rev. Lett. 5 (1960) 3.

[53] D. Glavan, S.P. Miao, T. Prokopec and R.P. Woodard, Graviton loop corrections to vacuum polarization in de Sitter in a general covariant gauge, Class. Quant. Grav. 32 (2015) 195014 [arXiv: 1504.00894] [INSPIRE].

[54] N.C. Tsamis and R.P. Woodard, Quantum gravity slows inflation, Nucl. Phys. B 474 (1996) 235 [hep-ph/9602315] [INSPIRE].

[55] N.C. Tsamis and R.P. Woodard, The quantum gravitational back reaction on inflation, Annals Phys. 253 (1997) 1 [hep-ph/9602316] [INSPIRE].

[56] N.C. Tsamis and R.P. Woodard, One loop graviton selfenergy in a locally de Sitter background, Phys. Rev. D 54 (1996) 2621 [hep-ph/9602317] [INSPIRE].

[57] N.C. Tsamis and R.P. Woodard, Dimensionally regulated graviton 1-point function in de Sitter, Annals Phys. 321 (2006) 875 [gr-qc/0506056] [InSPIRE].

[58] S.-P. Miao and R.P. Woodard, The fermion self-energy during inflation, Class. Quant. Grav. 23 (2006) 1721 [gr-qc/0511140] [INSPIRE].

[59] S.P. Miao and R.P. Woodard, Gravitons enhance fermions during inflation, Phys. Rev. D 74 (2006) 024021 [gr-qc/0603135] [INSPIRE].

[60] E.O. Kahya and R.P. Woodard, Quantum gravity corrections to the one loop scalar self-mass during inflation, Phys. Rev. D 76 (2007) 124005 [arXiv:0709.0536] [INSPIRE].

[61] E.O. Kahya and R.P. Woodard, Scalar field equations from quantum gravity during inflation, Phys. Rev. D 77 (2008) 084012 [arXiv:0710.5282] [InSPIRE].

[62] H. Kitamoto and Y. Kitazawa, Soft gravitons screen couplings in de Sitter space, Phys. Rev. D 87 (2013) 124007 [arXiv:1203.0391] [INSPIRE].

[63] H. Kitamoto and Y. Kitazawa, Soft graviton effects on gauge theories in de Sitter space, Phys. Rev. D 87 (2013) 124004 [arXiv: 1204.2876] [INSPIRE].

[64] H. Kitamoto and Y. Kitazawa, Scheme dependence of quantum gravity on de Sitter background, Nucl. Phys. B 873 (2013) 325 [arXiv:1211.3878] [InSPIRE].

[65] K.E. Leonard and R.P. Woodard, Graviton corrections to vacuum polarization during inflation, Class. Quant. Grav. 31 (2014) 015010 [arXiv: 1304.7265] [INSPIRE].

[66] H. Kitamoto and Y. Kitazawa, Soft gravitational effects in Kadanoff-Baym approach, JHEP 10 (2013) 145 [arXiv: 1305.2029] [INSPIRE].

[67] D. Glavan, S.P. Miao, T. Prokopec and R.P. Woodard, Electrodynamic effects of inflationary gravitons, Class. Quant. Grav. 31 (2014) 175002 [arXiv:1308.3453] [INSPIRE]. 
[68] H. Kitamoto and Y. Kitazawa, Time dependent couplings as observables in de Sitter space, Int. J. Mod. Phys. A 29 (2014) 1430016 [arXiv:1402.2443] [INSPIRE].

[69] C.L. Wang and R.P. Woodard, Excitation of photons by inflationary gravitons, Phys. Rev. D 91 (2015) 124054 [arXiv: 1408.1448] [INSPIRE].

[70] C.-S. Chu and Y. Koyama, Dilaton, screening of the cosmological constant and IR-driven inflation, JHEP 09 (2015) 024 [arXiv: 1506.02848] [INSPIRE]. 